\title{
Event Horizons in Static Scalar-Vacuum Space-Times
}

\author{
J. E. CHASE \\ Department of Mathematics, University of Alberta, Edmonton, Alberta, Canada
}

Received August 3, 1970

\begin{abstract}
The following theorem is established. Every zero-mass scalar field which is gravitationally coupled, static and asymptotically flat, becomes singular at a simplyconnected event horizon. In the special case where the gravitational coupling of the scalar energy density is neglected, the solutions are computed explicitly. Some properties of the singular event horizons are discussed, and a brief mention of related work with non-static scalar fields is given.
\end{abstract}

\section{Introduction}

Recent interest in the theory of gravitational collapse has raised many questions regarding the existence and nature of event horizons in relativity. Some definite results are known. Israel has shown [1] that for the class of asymptotically flat, static vacuum fields, only the spherically symmetric Schwarzschild solutions with $m \geqq 0$ have a regular event horizon $(r=2 m)$, and [2] that for the corresponding electrovac space-times, the Reissner-Nordström solutions with $m \geqq G^{\frac{1}{2}}|e| / c$ are the only ones with non-singular horizons. In view of these special cases, it is therefore natural to ask whether, for arbitrary, asymptotically flat static fields, a regular event horizon is destroyed by any asymmetric perturbation due to sources within the surface $g_{00}=0$.

In this connection there has been some recent interest in another special class - namely, the static coupled gravitational and massless scalar fields (where by "massless scalar field" we mean a scalar field for zero-mass particles). The spherically symmetric solution of Janis, Newman, and Winicour (JNW) [3] has the interesting property that the event horizon is a singular point in the space no matter how small the coupling constant becomes. Penney [4] has suggested that this surprising result is due to the imposition of spherical symmetry, and that, by considering asymmetric solutions, one is led to a nonsingular horizon. However, his example in support of this contention contains an error [5], and, in fact, his solution is singular at the horizon.

In this paper we propose to clear up much of the controversy about event horizons associated with asymptotically flat, static, massless scalar 
fields interacting with Einstein fields. Our main result can be stated as a theorem: every zero-mass scalar field which is gravitationally coupled, static and asymptotically flat, becomes singular at a simply-connected event horizon. This theorem immediately obviates Penney's search for a nonsingular asymmetric horizon.

We proceed by reformulating the given conditions in terms of the geometry of the surfaces $g_{00}=$ const. (Sec. 2,3). The theorem is stated in detail (Sec. 4) and proved (Sec. 5). In the special case where we neglect the gravitational effect of the scalar energy density, the solutions are computed explicitly (Sec.6). Some properties of the singular horizons are discussed, and a brief mention of some related results for non-static scalar fields is given (Sec. 7).

\section{Static Fields}

This section deals with the general static field. We want to reformulate the Einstein equations as conditions on the geometry of the equipotential surfaces. This has already been done in detail in Ref. [2], and need not be repeated here, except to list those formulas we will actually use.

The signature of the metric is -+++ . Capitalized Latin indices run from 0 to 3 . Three-dimensional and two-dimensional subtensors are distinguished by Greek indices (range 1-3) and by lower-case Latin indices (range 2-3). Covariant differentiation with respect to the 3-dimensional and 2-dimensional metrics is denoted by a stroke and a semicolon, respectively.

A space-time is called "static" if it admits a regular vector field $\xi$ which satisfies Killing's equations. In the domain where $\xi_{A} \xi^{A}<0$, the metric can be put in the form

$$
d s^{2}=g_{a b}\left(V, \theta^{1}, \theta^{2}\right) d \theta^{a} d \theta^{b}+\left[\varrho\left(V, \theta^{1}, \theta^{2}\right)\right]^{2} d V^{2}-V^{2} d t^{2}
$$

Here, $V$ is defined by

$$
V=\left(-\xi_{A} \xi^{A}\right)^{\frac{1}{2}}
$$

and

$$
\varrho^{-1}=\left(V_{\mid \alpha} V^{\mid \alpha}\right)^{\frac{1}{2}}
$$

vanishes nowhere in the domain of interest (if the 2-spaces $V=$ const., $t=$ const. do not bifurcate), while $\theta^{1}, \theta^{2}$ are intrinsic coordinates for the equipotential 2-spaces. Let $\boldsymbol{n}$ be the unit vector normal to the equipotential surfaces, and $\boldsymbol{e}_{(a)}$ the tangential base-vectors associated with $\theta^{a}$. Then we have

$$
\left.\begin{array}{rl}
n^{\alpha} & =\varrho^{-1} \partial x^{\alpha}\left(V, \theta^{1}, \theta^{2}\right) / \partial V \\
e_{(a)}^{\alpha} & =\partial x^{\alpha}\left(V, \theta^{1}, \theta^{2}\right) / \partial \theta^{a}
\end{array}\right\}
$$


The triad $\left\{\boldsymbol{e}_{(a)}, \boldsymbol{n}\right\}$ spans the 3-space at each point, and the following decompositions are derivable from (1):

$\left.\begin{array}{rl}g^{\alpha \beta} & =g^{a b} e_{(a)}^{\alpha} e_{(b)}^{\beta}+n^{\alpha} n^{\beta} \\ V_{l \alpha \beta} & =\varrho^{-1} K_{a b} e_{\alpha}^{(a)} e_{\beta}^{(b)}-\varrho^{-2} \varrho_{; c}\left(e_{\alpha}^{(c)} n_{\beta}+e_{\beta}^{(c)} n_{\alpha}\right)-\varrho^{-3}(\partial \varrho / \partial V) n_{\alpha} n_{\beta} .\end{array}\right\}$

Here,

$$
K_{a b} \equiv \frac{1}{2} \varrho^{-1} \partial g_{a b} / \partial V
$$

is the extrinsic curvature of the 2-space $V=$ const., considered as imbedded in the 3 -space $t=$ const. From (6) we have the related formula

$$
\partial g^{\frac{1}{2}} / \partial V=g^{\frac{1}{2}} \varrho K
$$

where $g$ is the $2 \times 2$ determinant of $g_{a b}$ and $K=g^{a b} K_{a b}$ is twice the mean curvature.

The Einstein field equations

$$
G_{A B}=-8 \pi \gamma T_{A B},
$$

(where $\gamma$ is Newton's constant of gravitation divided by $c^{2}$ ) can now be decomposed, yielding

$$
\begin{gathered}
\frac{1}{2} g^{\alpha \beta} R_{\alpha \beta}=8 \pi \gamma T_{0}^{0}, \\
0=8 \pi \gamma T_{\alpha 0}, \\
G_{\alpha \beta}=-8 \pi \gamma T_{\alpha \beta}-V^{-1}\left(V_{\mid \alpha \beta}-V_{\mid \mu}^{\mid \mu} g_{\alpha \beta}\right) .
\end{gathered}
$$

The relativistic analogue of Poisson's equation is then given by

$$
V_{\mid \mu}^{\mid \mu}=4 \pi \gamma V\left(T_{\alpha}^{\alpha}-T_{0}^{0}\right) .
$$

Finally, we record the following expressions [2] for later use:

$$
\begin{gathered}
\varrho^{-2} \partial \varrho / \partial V=K-4 \pi \gamma V \varrho\left(T_{\alpha}^{\alpha}-T_{0}^{0}\right), \\
V^{-1} g^{-\frac{1}{2}} \partial\left(g^{\frac{1}{2}} V K_{a}^{b}\right) / \partial V \\
=-\varrho_{; a}^{; b}-\frac{1}{2} \varrho R \delta_{a}^{b}-8 \pi \gamma \varrho\left(T_{\alpha \beta} e_{(a)}^{\alpha} e_{(b)}^{\beta}-\frac{1}{2} T_{A}^{A} g_{a b}\right), \\
\frac{1}{2}\left(K_{a b} K^{a b}-K^{2}-R\right)=-8 \pi \gamma T_{\alpha \beta} n^{\alpha} n^{\beta}+\varrho^{-1} V^{-1} K, \\
\partial_{a} K-K_{a ; b}^{b}=-8 \pi \gamma T_{\alpha \beta} e_{(a)}^{\alpha} n^{\beta}+\varrho^{-2} V^{-1} \partial_{a} \varrho \\
\frac{1}{4} R_{A B C D} R^{A B C D}=G_{\alpha \beta} G^{\alpha \beta}+\varrho^{-2} V^{-2} K_{a b} K^{a b} \\
+2 \varrho^{-4} V^{-2} \varrho_{; a} \varrho^{; a}+\varrho^{-6} V^{-2}(\partial \varrho / \partial V)^{2} .
\end{gathered}
$$

Eqs. (6), (11), and (12) form a complete system for determining the evolution of $g_{a b}, \varrho, K_{a}^{b}$ as functions of $V$. Eqs. (13) and (14) are involutive 
constraints, i.e. if they are satisfied on one surface $V=$ const., they must be satisfied identically. The last equation gives the (invariant) square of the four-dimensional Riemann tensor.

\section{Static Massless Scalar Fields}

We now consider a static scalar field $\phi$ for zero-mass particles, with "scalar gradient" given by

$$
\mathfrak{\Im}_{A}=-(4 \pi)^{\frac{1}{2}} \partial_{A} \phi .
$$

Since $\phi$ is static we have $\varsigma_{0}=0$. The scalar equation $\square \phi=0$ can be written

$$
\left(-\operatorname{det} g_{A B}\right)^{-\frac{1}{2}} \partial_{\alpha}\left[\left(-\operatorname{det} g_{A B}\right)^{\frac{1}{2}} g^{\alpha \beta} \partial_{\beta} \phi\right]=0 .
$$

In view of (1) this can also be written as

$$
V^{-1} \partial\left(V g^{\frac{1}{2}} \psi\right) / \partial V=-\partial_{a}\left(\varrho g^{\frac{1}{2}} \phi^{; a}\right),
$$

with $\psi$ defined by

$$
\partial \phi / \partial V=\varrho \psi .
$$

For a massless scalar field the energy tensor is given by

$$
T_{A B}=\partial_{A} \phi \partial_{B} \phi-\frac{1}{2} g_{A B} g^{C D} \partial_{C} \phi \partial_{D} \phi,
$$

or from (16) we can write

$$
\begin{aligned}
4 \pi T_{0}^{0} & =-\frac{1}{2} \mathfrak{\Im}^{2}, \\
4 \pi T^{0 \alpha} & =0, \\
4 \pi T^{\alpha \beta} & =\mathfrak{\Xi}^{\alpha} \mathfrak{\Im}^{\beta}-\frac{1}{2} g^{\alpha \beta} \mathfrak{\Xi}^{2},
\end{aligned}
$$

with

$$
\begin{aligned}
& \mathfrak{S}_{\alpha}=-(4 \pi)^{\frac{1}{2}}\left(\psi n_{\alpha}+e_{\alpha}^{(a)} \partial_{a} \phi\right), \\
& \mathfrak{\Xi}^{2} \equiv \mathfrak{S}_{\alpha} \mathfrak{\Xi}^{\alpha}=4 \pi\left(\psi^{2}+\phi_{; a} \phi^{; a}\right) .
\end{aligned}
$$

If we now substitute (21) into the basic Eqs. (11)-(14) of the previous section, we are led to the following complete first-order system for determining the $V$-dependence of $g_{a b}, \phi, \psi, \varrho$ and $K_{a}^{b}$ :

Geometrical equation

$$
\partial g_{a b} / \partial V=2 \varrho K_{a b} .
$$

Static scalar equations

$$
\begin{aligned}
\partial \phi / \partial V & =\varrho \psi, \\
\partial\left(V g^{\frac{1}{2}} \psi\right) / \partial V & =-V \partial_{a}\left(\varrho g^{\frac{1}{2}} \phi^{; a}\right),
\end{aligned}
$$


Gravitational equations

$$
\begin{aligned}
\varrho^{-2} \partial \varrho / \partial V & =K, \\
V^{-1} g^{-\frac{1}{2}} \partial\left(g^{\frac{1}{2}} V K_{a}^{b}\right) / \partial V & =-\varrho_{; a}^{; b}-\frac{1}{2} \varrho R \delta_{a}^{b}-8 \pi \gamma \varrho \phi_{; a} \phi^{; b},
\end{aligned}
$$

Involutive constraints

$$
\begin{aligned}
\frac{1}{2}\left(K_{a b} K^{a b}-K^{2}-R\right) & =-4 \pi \gamma\left(\psi^{2}-\phi_{; a} \phi^{; a}\right)+\varrho^{-1} V^{-1} K, \\
\partial_{a} K-K_{a ; b}^{b} & =-8 \pi \gamma \psi \phi_{; a}+\varrho^{-2} V^{-1} \varrho_{; a} .
\end{aligned}
$$

The following result, which we will need later, is obtained by contracting (24) and eliminating $R$ by means of (25):

$$
V \partial\left(V^{-1} K\right) / \partial V=-\varrho_{; a}^{; a}-\frac{1}{2} \varrho K^{2}-\varrho \Lambda_{a b} \Lambda^{a b}-8 \pi \gamma \varrho \psi^{2},
$$

where

$$
\Lambda_{a b}=K_{a b}-\frac{1}{2} g_{a b} K
$$

is a measure of deviation from spherical symmetry.

Finally, we combine (15) and (23) to obtain

$$
\begin{aligned}
\frac{1}{4} R_{A B C D} R^{A B C D} & =G_{\alpha \beta} G^{\alpha \beta}+\varrho^{-2} V^{-2} K_{a b} K^{a b} \\
& +2 \varrho^{-4} V^{-2} \varrho_{; a} \varrho^{; a}+\varrho^{-2} V^{-2} K^{2} .
\end{aligned}
$$

Although an exact expression for the term $G_{\alpha \beta} G^{\alpha \beta}$ could be evaluated from (5), (9c), (10), (21), and (22), it is sufficient to note here that, of the terms of this expression (all positive), one will be

$$
48 \pi^{2} \gamma^{2}\left(\psi^{2}+\phi_{; a} \phi^{; a}\right)^{2} \text {. }
$$

\section{Statement of Theorem}

In a static space-time, let $\Sigma$ be any spatial hypersurface $t=$ const., maximally extended consistent with $\xi_{A} \xi^{A}<0$. We consider the class of static massless scalar fields such that the following conditions are satisfied on $\Sigma$ :

(i) $\Sigma$ is a "scalar-vacuum" space (i.e. free of matter and sources of scalar fields).

(ii) $\Sigma$ is regular, non-compact and "asymptotically Euclidean". That is, there exist coordinates $x^{\alpha}$ in terms of which the metric (1) has the asymptotic form

$$
\left.\begin{array}{rl}
g_{\alpha \beta} & =\delta_{\alpha \beta}+0\left(r^{-1}\right), \partial_{\gamma} g_{\alpha \beta}=0\left(r^{-2}\right) \\
V & =1-(m / r)+\eta, m=\text { const. } \\
\eta & =0\left(r^{-2}\right), \partial_{\alpha} \eta=0\left(r^{-3}\right), \partial_{\alpha} \partial_{\beta} \eta=0\left(r^{-4}\right)
\end{array}\right\} \quad(r \rightarrow \infty)
$$

where $r=\left(\delta_{\alpha \beta} x^{\alpha} x^{\beta}\right)^{\frac{1}{2}}$. 
(iii) The asymptotic form of the static scalar field is

$$
\left.\begin{array}{l}
\phi=(k / r)+\zeta, \quad k=\text { const. } \\
\zeta=0\left(r^{-2}\right), \quad \partial_{\alpha} \zeta=0\left(r^{-3}\right)
\end{array}\right\} \quad(r \rightarrow \infty) .
$$

(iv) The equipotential surfaces $V=$ const. $>0, t=$ const., are a regular family of simply-connected, closed 2-spaces.

(v) If the greatest lower bound of $V$ on $\Sigma$ is zero, then the geometry of the equipotential surfaces $V=\varepsilon$ approaches a limit as $\varepsilon \rightarrow 0_{+}$, corresponding to a closed regular horizon of non-infinite area.

(vi) The invariant $R_{A B C D} R^{A B C D}$ is bounded on $\Sigma$.

Theorem. There is no non-trivial static space-time which satisfies conditions (i)-(vi).

(Here we assume $\gamma>0$. The case of zero coupling is discussed in Sec. 6.)

The proof of the theorem is presented in Section 5. There is one trivial case, however, which can be quickly disposed of here. Suppose that $V$ has a positive lower bound. Then $\Sigma$ is complete, hence spacetime is flat with $\phi \equiv 0[2]$.

We may assume henceforth that $V$ comes arbitrarily close to zero on $\Sigma$. The equipotential surface $V=0_{+}$then forms an inner boundary of $\Sigma$.

We conclude this section by recording the exterior and interior boundary conditions in a form convenient for later application. For the asymptotic forms (31) and (32), we find from (2), (7), and (19)

$$
\left.\begin{array}{l}
r \rightarrow \infty, \varrho / r^{2} \rightarrow m^{-1}, r k \rightarrow 2 \\
r \phi \rightarrow k, r^{2} \psi \rightarrow-k, \text { as } V \rightarrow 1
\end{array}\right\}
$$

According to (vi) and (29), the regularity of the manifold at the inner boundary $V=0_{+}$requires that

$$
K_{a b}=0(\varrho V), \quad \varrho_{; a}=0\left(\varrho^{2} V\right) \quad \text { as } \quad V \rightarrow 0_{+}
$$

It follows that $\varrho^{-1}$ is constant on the event horizon:

$$
\varrho^{-1}\left(0, \theta^{1}, \theta^{2}\right)=1 / \varrho_{0}=\text { const. }
$$

In addition, since the curvature scalar $R_{A B C D} R^{A B C D}$ is bounded everywhere on $\Sigma$ by (vi), it follows that the expression (30) cannot become infinite anywhere on $\Sigma$. Hence we have the result that $\psi$ and $\phi_{; a}$ are bounded functions throughout $\Sigma$. 


\section{Proof of Theorem}

In this section we employ mainly the scalar equations (18) and (19) to derive integral relations which enable us to show that the trivial solution $\phi \equiv 0$ is the only one compatible with conditions (i)-(vi).

Let $F(V, \phi), G(V, \phi)$ be (for the moment, arbitrary) differentiable functions. From (18), (19), (23), and (7) we easily obtain the identity $g^{-\frac{1}{2}}(\partial / \partial V)\left[g^{\frac{1}{2}}\left\{V F(V, \phi) \psi+\varrho^{-1} G(V, \phi)\right\}\right]$ $=A(V, \phi) \varrho\left(\psi^{2}+\phi_{; a} \phi^{; a}\right)+B(V, \phi) \psi+\varrho^{-1} \partial G / \partial V-V g^{-\frac{1}{2}}\left(F \varrho g^{\frac{1}{2}} \phi^{; a}\right)_{; a}$ where

$$
A \equiv V \partial F / \partial \phi, \quad B \equiv V \partial F / \partial V+\partial G / \partial \phi .
$$

In order to obtain integral conservation laws from (36), we require that

$$
A=B=\partial G / \partial V=0 .
$$

The general solution of this linear system of differential equations for $F, G$ is a linear combination of the two particular solutions

$$
\begin{array}{ll}
F=1, & G=1, \\
F=\ln V, & \mathrm{G}=-\phi .
\end{array}
$$

Taking these values in turn we integrate (36a) over $\Sigma$, i.e. we form $\iiint_{\Sigma}$ (36a) $g^{\frac{1}{2}} d V d \theta^{1} d \theta^{2}$, noting that the integral of the last term, being a 2-divergence, vanishes when taken over any closed 2-space $V=$ const. The results express the equality of the surface integrals of the expression in square brackets above over any two equipotential surfaces $V=$ const.:

$$
\begin{aligned}
\int\left(V \psi+\varrho^{-1}\right) d S & =C_{1}, \\
\int\left[(V \ln V) \psi-\varrho^{-1} \phi\right] d S & =C_{2},
\end{aligned}
$$

where we have defined the element of area by $d S=g^{\frac{1}{2}} d \theta^{1} d \theta^{2}$.

As an immediate consequence of (7) and (23) we have

$$
\partial\left(\varrho^{-1} g^{\frac{1}{2}}\right) / \partial V=0
$$

hence if we form $\iiint(40) d V d \theta^{1} d \theta^{2}$, we obtain

$$
\int \varrho^{-1} d S=C_{3} .
$$

Comparison of (39a) and (41) shows that

$$
\int V \psi d S=C_{1}-C_{3}=C_{4} .
$$

We can now evaluate the constants $C_{2}, C_{3}$, and $C_{4}$ by integrating ( $39 \mathrm{~b}$ ), (41), and (42) over the upper boundary $V=1$, with the help of the 
boundary conditions (33). We thus find, as integral conditions on the lower boundary $V=0_{+}$:

$$
\begin{gathered}
\int_{V=0_{+}} \varrho^{-1} d S=4 \pi m, \\
\int_{V=0_{+}} V \psi d S=-4 \pi k, \\
\int_{V=0_{+}}\left[(V \ln V) \psi-\varrho^{-1} \phi\right] d S=0 .
\end{gathered}
$$

In view of (35) we can write (43a) as

$$
S_{0} / \varrho_{0}=4 \pi m
$$

where $S_{0}$ is the area of the horizon $V=0_{+}$. Since $S_{0}$ is non-infinite by (v), (44) implies that $\varrho_{0}$ is also non-infinite.

We now consider the relation ( $43 \mathrm{~b}$ ). Since $\psi$ and the surface area are bounded on the horizon $V=0_{+}$, it follows that $k \equiv 0$, i.e.

$$
\int_{V=0+} V \psi d S=0 .
$$

In addition, the boundedness of $\varrho \psi=\partial \phi / \partial V$ and $\phi_{: a}$ throughout $\Sigma$ guarantees that $\phi$ itself is bounded on the horizon. This fact, together with (45) leads to

$$
\int_{V=0_{+}} V \phi \psi d S=0 .
$$

We now return to the identity (36) and this time require

$$
A=V, \quad B=\partial G / \partial V=0 .
$$

The resulting linear differential equations have the particular solution

$$
\begin{aligned}
& F=\phi+\ln V, \\
& G=-\phi .
\end{aligned}
$$

With $F, G$ given by (48), we thus have the identity

$$
\begin{aligned}
g^{-\frac{1}{2}}(\partial / \partial V)\left[g^{\frac{1}{2}}(V F \psi\right. & \left.\left.+\varrho^{-1} G\right)\right] \\
& =\varrho V\left(\psi^{2}+\phi_{; a} \phi^{; a}\right)-V g^{-\frac{1}{2}}\left(F \varrho g^{\frac{1}{2}} \phi^{; a}\right)_{; a} .
\end{aligned}
$$

Integrating over $\Sigma$ (again the last term doesn't contribute) we deduce the inequality

$$
\int_{V=1}\left(V F \psi+\varrho^{-1} G\right) d S \geqq \int_{V=0_{+}}\left(V F \psi+\varrho^{-1} G\right) d S .
$$

21 Commun math Phys., Vol 19 
From (49) it is clear that equality in (50) holds if and only if

$$
\left.\begin{array}{rl}
\psi & \equiv 0 \\
\phi_{; a} & \equiv 0
\end{array}\right\}
$$

everywhere on $\Sigma$. Now the surface integrals in (50) can actually be evaluated. The left side yields the value zero when integrated with the help of (33), as does the right side, in view of (43c) and (46). We conclude that (51) must hold, hence $\phi=$ const. throughout $\Sigma$. The fact that $\phi$ vanishes on the outer boundary $V=1$ ensures that $\phi \equiv 0$ and this completes the proof. It is worth noting that the theorem holds even for a regular point or line horizon $\left(S_{0}=0\right)$.

\section{Zero Coupling}

We consider in this section the case where the gravitational coupling of the scalar energy density is neglected. Our problem is to obtain solutions of the vacuum equations $G_{A B}=0$ and the scalar equation $\square \phi=0$, which satisfy conditions (i)-(vi) of Section 4. It is already known [1] that the only vacuum space-times compatible with (i)-(vi) are the Schwarzschild solutions with $m \geqq 0$. The problem thus reduces to finding well-behaved static scalar fields defined on the Schwarzschild background:

$$
\begin{aligned}
g_{a \beta} d x^{\alpha} d x^{\beta} & =(1-2 m / r)^{-1} d r^{2}+r^{2}\left(d \theta^{2}+\sin ^{2} \theta d \Phi^{2}\right), \\
\mathrm{V} & =(1-2 m / r)^{\frac{1}{2}} .
\end{aligned}
$$

The static scalar equation, $\square \phi=0$, reduces to

$$
\frac{V^{2}}{r^{2}} \frac{\partial}{\partial r}\left(V^{2} r^{2} \frac{\partial \phi}{\partial r}\right)+\frac{V^{2}}{r^{2} \sin \theta} \frac{\partial}{\partial \theta}\left(\sin \theta \frac{\partial \phi}{\partial \theta}\right)+\frac{V^{2}}{r^{2} \sin ^{2} \theta} \frac{\partial^{2} \phi}{\partial \Phi^{2}}=0 .
$$

Separable solutions which are regular on the axis have the form

$$
\phi=R(r) P_{n}^{M}(\cos \theta) e^{i M \Phi},
$$

where $R$ satisfies

$$
\begin{gathered}
\left(1-x^{2}\right) \frac{d^{2} R}{d x^{2}}-2 x \frac{d R}{d x}+n(n+1) R=0, \\
x=\frac{r}{m}-1 .
\end{gathered}
$$

For general $n,(55)$ has the linearly independent solutions

$$
R=C_{1} \mathfrak{P}_{n}(x), \quad R=C_{2} \mathfrak{Q}_{n}(x) .
$$


Of these solutions, the latter are unacceptable for all $n$, since $\mathfrak{Q}_{n}(x)$ is singular at $x=1$ (i.e. at the event horizon $r=2 m$ ). On the other hand, the former solutions have the wrong behaviour at infinity, except when $n=0$. Hence the only well-behaved solution satisfying (i)-(vi) occurs for $n=M=0$ and is given by

$$
\phi=C_{1}=0,
$$

from the boundary condition (31). We conclude that the trivial solution $\phi=0$ is the only static scalar field on a Schwarzschild background which is well-behaved for $2 m \leqq r<\infty$.

\section{Singular Point Horizons}

It is clear from the previous theorem that a non-trivial static scalarvacuum field must have a singular event horizon (be it a point or otherwise), and thus Penney's search [4] for an asymmetric solution with a regular event horizon is unnecessary. Our theorem also generalizes the work of JNW [3] and Gautreau [6] who show that all spherically symmetric solutions, and a certain class of axially symmetric solutions, respectively, always have singular horizons.

In the case of spherical symmetry, Eqs. (18) and (27) become simply

$$
\begin{gathered}
\partial\left(V g^{\frac{1}{2}} \psi\right) / \partial V=0, \\
V \partial\left(V^{-1} K\right) / \partial V=-\frac{1}{2} \varrho K^{2}-8 \pi \gamma \varrho \psi^{2} .
\end{gathered}
$$

Now (58), (40), and (19) can be solved explicitely with the help of the asymptotic forms (33) to give

$$
\phi=-k m^{-1} \ln V,
$$

which is a function of the two parameters $k$ and $m$. Consideration of (23), (59), and (60) enables us to solve for $\varrho$ and $K$ as functions of $V$, from which it can be determined that $\varrho \rightarrow 0$ as $V \rightarrow 0_{+}$. From (43a) it then follows that the area of the inner boundary $V=0_{+}$is zero, i.e. the horizon is a point (the non-regularity of the event horizon means that (35) is no longer true, but the integral condition (43a) still holds). This solution is precisely the JNW [3] solution ${ }^{1}$, which has a singular point horizon regardless of how small the coupling constant becomes. $r_{0}=2 m$,

${ }^{1}$ In our solution $k, m$, and $V$ are related to the $A, r_{0}, R$, and $\mu$ of JNW by: $A=-k / \sqrt{2}$,

$$
V=\left[\frac{2 R-r_{0}(\mu-1)}{2 R+r_{0}(\mu+1)}\right]^{\frac{1}{2 \mu}}, \quad \mu=\left(1+4 \pi \gamma k^{2} / m^{2}\right)^{\frac{1}{2}} \geqq 1 .
$$


The fact that the horizon is always a point in the case of spherical symmetry leads us to ask whether the same is true in general. Penney's example [4], although not regular as he had thought [5], serves to show that there are asymmetric solutions whose horizons, while singular, are not pointlike. His axially symmetric solution has the line element

$d s^{2}=e^{2 v}\left[\left(\frac{R}{R-2 m}\right) d R^{2}+R^{2} d \theta^{2}\right]+R^{2} \sin ^{2} \theta d \Phi^{2}-\left(\frac{R-2 m}{R}\right) d t^{2}$,

with

$$
v=-2 \pi \gamma a^{2} \frac{R(R-2 m) \sin ^{2} \theta}{\left[R(R-2 m)+m^{2} \cos ^{2} \theta\right]^{2}} .
$$

It is easy to check that the horizon $(R=2 m)$ is not pointlike. This example, along with (43a) suffices to show that the function $\varrho$ need not vanish everywhere on the horizon $V=0_{+}$. We can, however, show for a general scalar solution with event horizon of bounded area, that $\varrho$ always vanishes at least locally there. From (23) and (40) it is easily shown that

$$
\frac{d}{d V} \int \varrho^{-2} d S=-\int \varrho^{-1} K d S .
$$

From (27) and (40) we have

$$
\begin{aligned}
\partial\left(\varrho^{-1} g^{\frac{1}{2}} K\right) / \partial V= & \varrho^{-1} V^{-1} g^{\frac{1}{2}} K-8 \pi \gamma g^{\frac{1}{2}} \psi^{2}-\frac{1}{2} g^{\frac{1}{2}} K^{2} \\
& -g^{\frac{1}{2}}\left[(\ln \varrho) ; a+\varrho^{-2} \varrho_{; a} \varrho^{; a}+\Lambda_{a b} \Lambda^{a b}\right] .
\end{aligned}
$$

If we now form $\iiint(64) d V d \theta^{1} d \theta^{2}$ and use the Schwarz inequality on the second term of the right side,

$$
\int \psi^{2} d S \geqq \frac{1}{S}\left[\int \psi d S\right]^{2},
$$

it is straightforward to arrive at the inequality

$$
\frac{d}{d V} \int K Q^{-1} d S \leqq \frac{1}{V} \int K \varrho^{-1} d S-\frac{8 \pi \gamma}{S}\left[\int \psi d S\right]^{2},
$$

which, in view of (42) can be written

$$
\frac{d}{d V} \int K \varrho^{-1} d S \leqq \frac{1}{V} \int K \varrho^{-1} d S-\frac{\text { const. }}{V^{2} S} .
$$

If we now let

$$
X(V)=\int \varrho^{-2} d S,
$$

then (63) and (66) combine to give

$$
\frac{d}{d V}\left[\frac{1}{V} \frac{d X}{d V}\right] \geqq \frac{\text { const. }}{V^{3} S} .
$$


Since $S=S(V)$ is a continuous function, infinite only on the outer boundary $V=1$, then for any $V_{1}<1, S(V)$ must attain a maximum $S_{M}$ somewhere in the interval from $V=0_{+}$to $V=V_{1}$. In this interval we thus have $1 / S \geqq 1 / S_{M}$, hence

$$
\frac{d}{d V}\left[\frac{1}{V} \frac{d X}{d V}\right] \geqq \frac{\text { const. }}{V^{3}} .
$$

Integrating (69) twice we arrive at the inequality

from which

$$
X(V) \geqq-C_{1} \ln V+C_{2} V^{2}+C_{3},
$$

$$
X(0)=\int_{V=0_{+}} \varrho^{-2} d S=\infty .
$$

The integral condition (71) along with ( 43 a) shows that $\varrho$ becomes zero at least locally on the inner boundary $V=0_{+}$(this reaffirms that for spherical symmetry the horizon must be a point). It is also clear from (43a) that the region of the horizon on which $\varrho$ vanishes must be of zero area. In other words the gravitational flux $\varrho^{-1}=|\nabla V|$ becomes infinite somewhere on the horizon because some finite-area flux tube shrinks to a point or line there.

In the case of Penney's example, comparison of (1) and (61) shows

$$
\begin{gathered}
V=\left(\frac{R-2 m}{R}\right)^{\frac{1}{2}}, \\
\varrho^{2} d V^{2}=e^{2 v}\left(\frac{R}{R-2 m}\right) d R^{2} .
\end{gathered}
$$

From (72) and (73) we immediately deduce that

$$
\varrho=e^{v} R^{2} / m .
$$

On the horizon $R=2 m$, consideration of (62) shows that $\varrho$ vanishes in the equatorial plane $\theta=\pi / 2$.

We can summarize our results for static scalar fields as follows:

(1) Every massless scalar field which is gravitationally coupled, static and asymptotically flat, becomes singular at a simply-connected event horizon.

(2) In the case of spherical symmetry, the singular horizons are points.

(3) If we allow asymmetric solutions, it is possible to find examples for which the horizon is not pointlike.

(4) Assuming the horizon has bounded surface area, the gravitational flux $\varrho^{-1}$ always becomes singular somewhere on the horizon, due to the shrinking of some finite-area flux tube to a point or line. 
Finally, we mention some recent investigations into non-static scalar fields. Qualitative considerations by Israel [7] have indicated that, if such a field is anchored to a collapsing star, some of the field energy will drain downwards through the event horizon $(r=2 m)$ on a time scale $t \sim m$ as measured by a distant observer. Taking as a model a highly idealized star collapsing with the speed of light, detailed numerical integrations have been carried out which confirm these considerations [8]. We find that the external field decays in an oscillatory fashion. The energy lost goes partly into outflowing radiation, but most of it (about $68 \%$ ) falls in through the horizon after the star has collapsed. The oscillatory nature of the decay has been described by Israel [7] as a "bathplug effect": blobs of field energy drawn inwards by gravity cannot be sucked in all at once, but are first turned aside by field pressure and swing about the hole on a time-scale several times larger than the Schwarzschild characteristic time $2 m$ (the factor is roughly 10 in our numerical results).

Acknowledgements. I wish to thank Professor W. Israel for suggesting the problem, and for many helpful discussions and suggestions during the work. I would also like to acknowledge the financial support of the National Research Council of Canada.

\section{References}

1. Israel, W.: Phys. Rev. 164, 1776 (1967).

2. - Commun. Math. Phys. 8, 245 (1968).

3. Janis, A. I., Newman, E. T., Winicour, J.: Phys. Rev. Letters 20, 878 (1968) [as corrected by Janis, Newman, Winicour: Phys. Rev. 176, 1507 (1968)].

4. Penney, R.: Phys. Rev. 174, 1578 (1968).

5. Janis, A. I., Robinson, D. C., Winicour, J.: Phys. Rev. 186, 1729 (1969).

6. Gautreau, R.: Coupled Weyl gravitational and zero-rest-mass scalar fields (a preprint).

7. Israel, W.: GRG Vol. 1, No. 3 (to be published).

8. - Chase, J. E.: To be published.

J. E. Chase

Department of Mathematics

The University of Alberta

Edmonton, 7, Alberta, Canada 